\title{
Long-term spatiotemporal dynamics of solid-state lasers and vertical-cavity surface-emitting lasers
}

\author{
G.-L. Oppo* and A. M. Yao \\ Department of Physics and SUPA, University of Strathclyde, Glasgow G4 ONG, Scotland, United Kingdom \\ F. Prati \\ Dipartimento di Fisica e Matematica, CNISM and INFM-CNR, Università dell'Insubria, via Valleggio 11, I-22100 Como, Italy \\ G. J. de Valcárcel \\ Departament d'Òptica, Universitat de València, Dr. Moliner 50, E-46100 Burjassot, Spain \\ (Received 15 October 2008; published 17 March 2009)
}

\begin{abstract}
The number of Maxwell-Bloch equations describing the spatiotemporal evolution of solid-state and semiconductor-based lasers can be reduced when the temporal scales of the fields and atomic variables are very different. We demonstrate the existence of slow (center) manifolds for models of solid-state lasers (such as $\mathrm{Nd}$ :YAG) and vertical-cavity surface-emitting lasers and determine reduced equations in the presence of both diffraction and carrier diffusion. Two separate methods of obtaining the reduced equations are presented. Excellent agreement between the reduced models and the original equations is obtained when the slow manifolds are expanded in a perturbative series. Since stiffness is removed, the computational time for the long-term spatiotemporal dynamics of these devices can be strongly reduced, typically by 2 orders of magnitude.
\end{abstract}

DOI: 10.1103/PhysRevA.79.033824

PACS number(s): 42.65.Sf, 42.55.Px, 42.55.Rz, 05.10.-a

\section{INTRODUCTION}

Several numerical models based on Maxwell-Bloch (MB) equations for the spatiotemporal dynamics of solid-state (including semiconductor) lasers suffer from the so-called "stiffness." Stiffness corresponds to the fact that dynamical variables (here the optical field inside the cavity, the carrier density, and the dielectric polarization) evolve on very different time scales, so that numerical implementation is quite inefficient. Semiconductor laser modeling is far more complex than "standard" solid-state (e.g., based on solid-state doped materials such as Nd:YAG) device modeling. The problem of stiffness is, however, common to both these groups of lasers since in both cases the cavity field and, in particular, the carrier density evolve on time scales much longer than other dynamical variables such as the medium polarization.

Effective reduced models that eliminate stiffness can be of importance in reducing the computational time and hence increasing the predictive power of these numerical simulations. Stiffness in ordinary and partial differential equations can be eliminated by reducing the number of coupled variables to just those that evolve on a slow time scale. Several years ago, Ref. [1] showed that in laser models which include diffraction the straightforward elimination of fast variables can lead to spurious instabilities and that more advanced techniques are needed for a correct description of their long-term spatiotemporal dynamics. Moreover, the difficulty in obtaining simple reduced models for broad ranges of parameter values has often restricted the analysis to regimes very close to the laser threshold [2,3]. In recent years Maxwell-Bloch models have been used extensively in the

*gianluca@phys.strath.ac.uk simulation of spatiotemporal structures in broad area microcavity semiconductor absorbers [4] and in vertical-cavity surface-emitting lasers (VCSELs) [5]. Close-to-threshold models are excellent in capturing the essential ingredients for the dynamics of laser devices but, of course, cannot describe the output of these modern solid-state lasers that may be operated several times above threshold.

One method standardly used in the literature to eliminate stiffness is based on the theory of center manifolds (CMs) [6]. CMs are subspaces of the original phase space that attract the long-term dynamics of the system. They are also known as slow manifolds (SMs). One of the presumed limitations of center manifold theory is that the perturbation expansion is restricted to the neighborhood of a specific steadystate solution. In the laser problem, where there are at least two states of interest corresponding to on and off lasing action (and all their bifurcating solutions), it appeared that reductions based on center manifolds would possess a local character only [1]. Here we show that this difficulty can be circumvented by using higher-order expansions and linear operators. We derive and test reduced models for standard solid-state doped-crystal lasers and VCSELs. With typical decay times of the medium polarization, the cavity field and the carrier densities of around $100 \mathrm{fs}, 10 \mathrm{ps}$, and $1 \mathrm{~ns}$, respectively, a carefully reduced model can achieve the same asymptotic result as the original simulation 1000 times faster. Because of the larger number of operations per step required in the reduced model, we have achieved gain factors of 300 and more in the simulation times of the same spatial structures for both solid-state doped lasers and VCSELs. We also show that the agreement between the original and reduced models extends to lasers operated far above threshold where models derived under the close-tothreshold approximation can no longer be applied. For example, we show that a Hopf bifurcation with a finite trans- 
verse wave vector is absent in close-to threshold models but is accurately reproduced by our reduced equations.

The paper is organized as follows. In Sec. II we discuss the theory of CMs. We then demonstrate the existence of CMs containing more than one fixed point and with a broader range of validity than previously considered for the solid-state (Sec. III) and VCSEL (Sec. IV) models. We next apply a perturbative determination of the CM technique to obtain reduced equations. A further rescaling through a singular perturbation expansion of the population (carrier) variable is then applied. In particular, we show that the dynamics of the complex amplitude of traveling-wave solutions is accurately described by the reduced models at resonance. $\mathrm{Nu}$ merical comparisons demonstrating the wide range of validity and applications of the reduced models are presented in Sec. V. Conclusions and discussion of the relevance of our results to the analysis of dissipative spatiotemporal structures in the output of a large variety of laser systems are presented in Sec. VI.

\section{EXISTENCE OF SLOW (CENTER) MANIFOLDS}

In Ref. [1] CM techniques were applied to spatiotemporal models of lasers. For convenience the CM was developed around a specific stationary solution of the model equations. In the laser case there are at least two stationary solutions above threshold: one corresponding to lasing and one corresponding to nonlasing action. Without spatial effects only the lasing solution is relevant. In fact, the nonlasing solution lies on an unstable manifold that cannot be reached by the dynamical evolution of the differential equations since once it is attained it traps the system dynamics forever. For this reason the logarithmic transformation $s=\ln (I)$ with $I$ being the laser intensity was introduced in Ref. [7]. Such transformation moves the nonlasing fixed point corresponding to $I=0$ to minus infinity.

With spatial effects due to the inclusion of diffraction, the nonlasing solution loses stability to the homogeneous lasing solution for one sign of the detuning (say that corresponding to positive $\theta$ ). In this case the logarithmic transformation remains valid. For the opposite sign of the detuning, however, the zero solution loses stability to traveling-wave patterns [8]. Standing wave solutions, obtained from two counterpropagating traveling waves with suitable boundary conditions [9], alternate regions of zero and nonzero laser intensities and so the logarithmic transformation loses its validity. There thus appeared to be the need for two CMs for laser equations: one for positive and one for negative detunings (see Ref. [1]).

An elegant, yet partial, solution to the problem was provided by Lega et al. [2,10] while introducing a complex Swift-Hohenberg (CSH) equation for lasers. The CSH reduced model is, at least in principle, valid only in a rectangle of sides $\left(\epsilon, \epsilon^{2}\right)$ (where $\epsilon$ is the smallness parameter) in the (detuning, pump) parameter region (see Fig. 1). Within this parameter region, all relevant homogeneous solutions and phase modulated patterns are correctly described by the CSH equation. In the stiff limit, however, an effective equation for the medium population must be added to the model in order

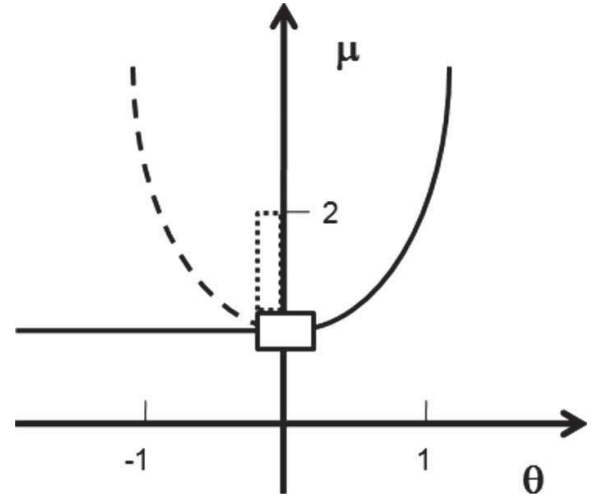

FIG. 1. Simplified detuning $\theta$ and pump $(\mu)$ parameter space for a standard laser. Without diffraction the parabola represents the laser threshold. With diffraction, traveling-wave patterns are generated for negative detunings at a flat threshold. The solid rectangle, of sides $\epsilon$ and $\epsilon^{2}$, represents the range of validity of the CSH model derived in [2] while the dashed rectangle represents the extended validity of a model formed by a CSH plus an effective equation for the population variable as described in [10].

to catch the instabilities of the traveling-wave solution that arise, in that limit, from the coupled dynamics of the electric field and the slow population. One remarkable fact of work of Lega et al. is that the reduced model formed by the CSH equation for the field and a mean flow equation for the population displayed agreement with the original equations well beyond the perturbative limit of the close-to-threshold approximation and up to twice the threshold value for negative detunings (see the dashed rectangle in Fig. 1). Such considerations have recently been extended to VCSEL models in [3].

It is the aim of this paper to obtain reduced models that maintain their validity far from threshold. The application of the CM theorem [6] guarantees the accuracy and validity of the final equations. Let us then briefly recall the main statement of the CM theorem: if a set of ordinary or partial differential equations for the vector variables $Z$ and $Y$ can be written in the form

$$
\begin{gathered}
\partial_{t} Z=A Z+\epsilon F(Z, Y, \epsilon), \\
\partial_{t} Y=\epsilon G(Z, Y, \epsilon),
\end{gathered}
$$

where $\epsilon$ is a smallness parameter and $A$ is a matrix with eigenvalues with negative real part, then a center manifold

$$
Z=H(\epsilon, Y)
$$

exists and can be found perturbatively. This implies that there are two distinct time scales for the evolution of the $Z$ and $Y$ variables. On the short time scale the $Z$ vector quickly relaxes to a submanifold of the full phase space. On the long-term time scale the dynamics is confined to the CM and will not leave such subregion of the phase space since the $\mathrm{CM}$ is asymptotically stable. Note that formula (2) is equivalent to a multiple time scale analysis. 
We first derive the dynamical equations evolving on the slow manifold and then extend the time scale by using a singular perturbation expansion of the population (carrier) variable $D$. These methods are applied to both solid-state and VCSEL models (Secs. III and IV, respectively).

\section{DERIVATION OF THE REDUCED EQUATIONS: THE SOLID-STATE LASER CASE}

We start with the standard Maxwell-Bloch equations for a solid-state doped laser (e.g., Nd:YAG) in the presence of diffractive effects. We define with $E$ and $P$ the complex slowly varying amplitudes of the electric and polarization fields, with $D$ as the normalized population, with $\epsilon$ and $\gamma\left(=\sigma \epsilon^{2}\right)$ as the decay rates of the field and population normalized to that of the polarization, with $\theta$ and $\mu$ as the detuning parameter and the amplitude of the external pump, and we use transverse space units such that the diffraction parameter, which is usually denoted by $a$, is equal to 1 . The resulting equations, after the mean-field limit, are $[1,11]$

$$
\begin{gathered}
\partial_{t} E=i \nabla^{2} E+\epsilon[P-(1+i \theta) E], \\
\partial_{t} P=-(1-i \theta) P+D E, \\
\partial_{t} D=-\sigma \epsilon^{2}\left[D-\mu+\operatorname{Re}\left(E^{*} P\right)\right],
\end{gathered}
$$

where the reference frequency is that of the spatially independent (homogeneous) single-mode lasing solution. Typical orders of magnitude of the normalized decay rates provide $\epsilon$ in the range of $10^{-2}-10^{-3}$ for a large variety of solid-state lasers, while the parameter $\sigma$ is of order 1 or smaller. We then consider $\epsilon$ as our smallness parameter and note the structure of the equations for $\epsilon=0$,

$$
\begin{gathered}
\left(\partial_{t}-i \nabla^{2}\right) E=0, \\
\partial_{t} P=-(1-i \theta) P+D E, \\
\partial_{t} D=0 .
\end{gathered}
$$

These equations admit a simple traveling-wave solution

$$
\begin{gathered}
E=A \exp [i(\vec{k} \cdot \vec{x}-\omega t)], \\
P=\frac{D E}{1-i \theta-i \omega}, \\
D=\text { const }
\end{gathered}
$$

where $A$ is a constant amplitude, $\vec{k}$ is the spatial wave vector, and $\omega$ satisfies the dispersion relation $\omega=k^{2}$.

If $\epsilon \neq 0$ the traveling-wave solution has the same form as Eq. (5) but with $A$ and $D$ given by

$$
\begin{gathered}
A=\sqrt{\mu-\mu_{\mathrm{th}}} e^{i \phi}, \quad D=\mu_{\mathrm{th}}, \\
\mu_{\mathrm{th}}=1+\left(\theta+\frac{k^{2}}{1+\epsilon}\right)^{2},
\end{gathered}
$$

$$
\omega=\frac{k^{2}}{1+\epsilon},
$$

where $\phi$ is an arbitrary phase and $\mu_{\text {th }}$ is the laser threshold. This illustrates the role of the sign of $\theta$ in the wave-vector selection at threshold: for $\theta<0$, the threshold has a minimum, $\mu_{\mathrm{th}}=1$, when the wave vector $\vec{k}$ is such that $k^{2}=-\theta(1$ $+\epsilon)$; for $\theta>0$, the threshold has a minimum, $\mu_{\mathrm{th}}=1+\theta^{2}$, for the homogeneous solution $\vec{k}=0$. Notice that $\theta(1+\epsilon)$ represents the detuning between the atomic frequency and the cavity frequency of the nearest longitudinal mode, while $\theta$ is the detuning between the atomic frequency and the lasing frequency of the nearest longitudinal mode. The latter is smaller by a factor $1+\epsilon$ which accounts for the mode pulling effect. In the presence of diffraction, the traveling-wave solution with the minimum threshold is that resonant with the atomic transition.

The polarization is still given by Eq. (5b) which, taking into account the above expression for $\omega$, can be rewritten as

$$
P=D \mathcal{L} E
$$

after using the operator $\mathcal{L}$ defined as

$$
\mathcal{L}=\left(1-i \theta+\frac{i \nabla^{2}}{1+\epsilon}\right)^{-1} \text {. }
$$

This suggests that a convenient way to recast Eq. (3) in the same form as Eq. (1) is to use a dynamical variable $Z$ and a space-time differential operator $\mathcal{D}$ defined as

$$
\begin{aligned}
& Z=P-D \mathcal{L} E, \\
& \mathcal{D}=\partial_{t}-\frac{i \nabla^{2}}{1+\epsilon} .
\end{aligned}
$$

We observe that both $\mathcal{D}$ and $\mathcal{L}$ are linear operators. The equations for $Z, E$, and $D$ are

$$
\begin{gathered}
\mathcal{D} Z=-\mathcal{L}^{-1} Z-\left(\partial_{t} D\right)(\mathcal{L} E)-D \mathcal{L}(\mathcal{D} E), \\
\mathcal{D} E=\epsilon(\mathcal{Q} E+Z),
\end{gathered}
$$

$$
\mathcal{D} D=-\sigma \epsilon^{2}\left\{D-\mu+\operatorname{Re}\left[E^{*}(D \mathcal{L} E+Z)\right]\right\}-\frac{i \epsilon \nabla_{\epsilon}^{2} D}{1+\epsilon},
$$

where the operator $\mathcal{Q}$ is defined as

$$
\mathcal{Q}=D \mathcal{L}-1-i \theta+\frac{i \nabla^{2}}{1+\epsilon} .
$$

In the equation for $D$ the substitution $\nabla^{2}=\epsilon \nabla_{\epsilon}^{2}$, with $\nabla_{\epsilon}^{2}$ $=\partial^{2} /\left(\epsilon \partial x^{2}\right)+\partial^{2} /\left(\epsilon \partial y^{2}\right)$, accounts for the fact that the population is constant in space and time for $\epsilon=0$ and hence we can assume that, in general, $D(t, x, y)=D(\epsilon t, \sqrt{\epsilon} x, \sqrt{\epsilon} y)$. This agrees with the phase modulations of traveling waves for the electric field and the polarization (see Eq. (5) and Refs. $[8,10,12])$.

Equation (11) admits a single center (slow) manifold for all traveling-wave solutions and for both signs of the detun- 
ing $\theta$ since all the terms on the right-hand sides (r.h.s's) of the second and third equations are on the order of $\epsilon$ or higher. Note that a CM of the Maxwell-Bloch equations is a subspace $(E, D)$ of the full phase space $(Z, E, D)$ where the longterm spatiotemporal dynamics takes place. The expression for $Z$ in the equations for $E$ and $D$ can be obtained perturbatively by expanding $Z$ in a power series of $\epsilon$, i.e.,

$$
Z=Z_{0}+\epsilon Z_{1}+\epsilon^{2} Z_{2}+O\left(\epsilon^{3}\right)
$$

and inserting it in Eq. (11a). Taking into account the definition of $\mathcal{D}$, the zeroth-order equation $\partial_{t} Z_{0}=-(1-i \theta) Z_{0}$ provides $Z_{0}=0$ on a long time scale. We observe that, should we stop at this level of approximation, the equations for $E$ and $D$ with $Z=0$, which read

$$
\begin{gathered}
\partial_{t} E=i \nabla^{2} E+\epsilon[D \mathcal{L} E-(1+i \theta) E], \\
\partial_{t} D=-\sigma \epsilon^{2}\left\{D\left[1+\operatorname{Re}\left(E^{*} \mathcal{L} E\right)\right]-\mu\right\},
\end{gathered}
$$

would contain enough information to exactly reproduce the traveling-wave solution of the full system, because for this solution $P=D \mathcal{L} E$. Hence, these equations already provide a good approximation of the full model. They can be also derived in an alternative way, as shown in Appendix A.

However, in order for the reduced model to better simulate the full model, including the stability of the traveling waves and the dynamical behavior of the system when those solutions become unstable, next-order corrections in $Z$ must be included. The first- and second-order terms in the perturbation expansion are

$$
Z_{1}=-\mathcal{L}[D \mathcal{L}(\mathcal{Q} E)]
$$

$$
\begin{aligned}
Z_{2}= & \sigma \mathcal{L}\left\{\left[D-\mu+\operatorname{Re}\left(E^{*} D \mathcal{L} E\right)\right](\mathcal{L} E)\right\}+\mathcal{L}\left\{D \mathcal{L}^{2}[D \mathcal{L}(\mathcal{Q} E)]\right\} \\
& +\mathcal{L}^{2}\left[D \mathcal{L}\left(\mathcal{Q}^{2} E\right)\right]+i \mathcal{L}\left(\frac{\partial Z_{1}}{\partial D} \nabla_{\epsilon}^{2} D\right) .
\end{aligned}
$$

Again splitting the operator $\mathcal{D}$ into its temporal and spatial components, the reduced equations in the subspace $(E, D)$ become

$$
\begin{gathered}
\partial_{t} E=\frac{i \nabla^{2} E}{1+\epsilon}+\epsilon(\mathcal{Q} E+Z) \\
\partial_{t} D=-\sigma \epsilon^{2}\left\{D-\mu+\operatorname{Re}\left[E^{*}(D \mathcal{L} E+Z)\right]\right\}
\end{gathered}
$$

with $Z=\epsilon Z_{1}+\epsilon^{2} Z_{2}$. It is clear that we can reduce the stiffness of the equations if in Eq. (16a) we can make the substitution $\nabla^{2}=\epsilon \nabla_{\epsilon}^{2}$ already used in Eq. (11c), i.e., if $E$ varies slowly in space. This assumption is certainly true if $|\theta|=O(\epsilon)$ because in that case the wave vector $\vec{k}$ of the traveling-wave solutions is such that $k^{2}=O(\epsilon)$. It is also true for $\theta$ large and positive if the homogeneous solution selected at threshold is either stable or unstable against small wave vectors. If $\theta$ is large and negative the traveling waves selected at threshold oscillate rapidly in space and time. Nevertheless, we can extract the rapidly oscillating terms from the electric field and write a set of equations with reduced stiffness for the slowly varying envelope. In the following we consider the different limits separately.

\section{A. $|\boldsymbol{\theta}|=\boldsymbol{O}(\boldsymbol{\epsilon})$}

In this limit we can set $\theta=\epsilon \delta$ with $\delta=O(1)$ and assume that $E$ varies slowly in space. With the substitution $\nabla^{2}=\epsilon \nabla_{\epsilon}^{2}$ the dynamical equations become

$$
\begin{gathered}
\partial_{\epsilon t} E=\frac{i \nabla_{\epsilon}^{2} E}{1+\epsilon}+\mathcal{Q} E+Z, \\
\partial_{\epsilon t} D=-\sigma \epsilon\left\{D-\mu+\operatorname{Re}\left[E^{*}(D \mathcal{L} E+Z)\right]\right\} .
\end{gathered}
$$

These equations evolve on a slower time scale $(\epsilon t)$ than the original $\mathrm{MB}$ equations and contain the long-term dynamics of the laser systems. From their structure, it may appear that a further reduction in the number of equations is possible since the rhs of the dynamical equation for the variable $D$ is multiplied by $\epsilon$. It has been shown, however, that in the limit of small $\epsilon$ the system approaches a conservative dynamics and that the reduction to a single variable $D$ is not possible [7]. Nevertheless, a renormalization of the temporal scales by a further singular expansion in $\sqrt{\epsilon}$ is still feasible. With this aim in mind we observe that in this limit $\mathcal{L}=1+O(\epsilon)$ and $\mathcal{Q}=D-1$ at order zero in $\epsilon$. Hence, if we use the following singular scaling for the population variable $[1,7]$

$$
D=1+\sqrt{\epsilon} W,
$$

and the time $\tau=\epsilon^{3 / 2} t$, the stiffness of the equations for $E$ and $W$ may be completely removed.

Taking into account that $\mathcal{L}=1+O(\epsilon)$ and $\mathcal{Q}=O(\sqrt{\epsilon})$ we can easily find the expression for $Z$ valid in this limit as follows:

$$
Z=-\epsilon^{3 / 2}\left[(1+\sqrt{\epsilon} W) W-\sigma \sqrt{\epsilon}\left(1-\mu+|E|^{2}\right)\right] E .
$$

Expanding $\mathcal{L}$ and $\mathcal{Q}$ in power series of $\epsilon$, the final equations for $E$ and $W$ are

$$
\begin{aligned}
\partial_{\tau} E= & \left\{\frac{i \nabla_{\epsilon}^{2}}{\sqrt{\epsilon}(1+\epsilon)}+\frac{W}{1+\epsilon}+i \epsilon W\left(\delta-\nabla_{\epsilon}^{2}\right)-\epsilon^{3 / 2}\left[W^{2}+\left(\delta-\nabla_{\epsilon}^{2}\right)^{2}\right.\right. \\
& \left.\left.-\sigma\left(1-\mu+|E|^{2}\right)\right]\right\} E
\end{aligned}
$$

$$
\begin{aligned}
\partial_{\tau} W= & -\sigma\left[(1+\sqrt{\epsilon} W)\left(1+|E|^{2}\right)-\mu-\epsilon(1+\sqrt{\epsilon} W) \operatorname{Re}\left(i E^{*} \nabla_{\epsilon}^{2} E\right)\right. \\
& \left.-\epsilon^{3 / 2} W|E|^{2}\right] .
\end{aligned}
$$

Notice that the term $\left(\delta-\nabla_{\epsilon}^{2}\right)^{2}$ in Eq. (20a) implies that the traveling-wave solution selected at threshold for negative $\delta$ is the one with wave vector $k^{2}=-\epsilon \delta=-\theta$ instead of $k^{2}=$ $-\theta(1+\epsilon)$, as predicted by the full model. However, in the limit $|\theta|=O(\epsilon)$ this results just in an error of the order $\epsilon^{3}$ in the intensity of the traveling-wave solution and in its threshold, while the frequency is still correct up to that order.

The close-to-threshold limit of Eq. (20) amounts to assuming that $D-1, \mu-1$, and $|E|^{2}$ are $O\left(\epsilon^{2}\right)$. The assumption on $D$ is equivalent to $W=O\left(\epsilon^{3 / 2}\right)$. The reduced equations valid in the close-to-threshold limit can be obtained from Eq. (20) keeping only terms up to the order $\epsilon^{3 / 2}$,

$$
\partial_{\tau} E=\frac{i \nabla_{\epsilon}^{2} E}{\sqrt{\epsilon}(1+\epsilon)}+W E-\epsilon^{3 / 2}\left(\delta-\nabla_{\epsilon}^{2}\right)^{2} E,
$$




$$
\partial_{\tau} W=-\sigma\left(1+\sqrt{\epsilon} W+|E|^{2}-\mu\right) .
$$

In Appendix B these equations are shown to be equivalent to those of Lega et al. [2,10] when the field decay rate of those equations is $O(\epsilon)$ as in our model.

$$
\text { B. }|\boldsymbol{\theta}|=\boldsymbol{O}(\mathbf{1})
$$

When the detuning $\theta$ is large and positive, the lasing solution above threshold is homogeneous in space $(k=0)$. If, instead, $\theta$ is large and negative the traveling wave with the minimum threshold oscillates rapidly in both time and space. In order to separate the fast and slow temporal and spatial scales we write the electric field $E$ as

$$
E(x, y, t)=F(\sqrt{\epsilon} x, \sqrt{\epsilon} y, \epsilon t) \exp \left[i\left(\vec{k} \cdot \vec{x}-\frac{k^{2}}{1+\epsilon} t\right)\right]
$$

and observe that we can write

$$
\nabla^{2} E=e^{i \vec{k} \cdot \vec{x}}\left(-k^{2}+2 i \sqrt{\epsilon} \vec{k} \cdot \vec{\nabla}_{\epsilon}+\epsilon \nabla_{\epsilon}^{2}\right) F
$$

where $\vec{\nabla}_{\epsilon}$ is the gradient operator in the plane $(\sqrt{\epsilon} x, \sqrt{\epsilon} y)$. It follows that $\mathcal{L} E=e^{i \vec{k} \cdot \vec{x}} \mathcal{L}^{\prime} F$, where the operator $\mathcal{L}^{\prime}$ acts only on the slowly varying amplitude $F$ and is defined as

$$
\mathcal{L}^{\prime}=\left(1-i \Delta-\frac{2 \sqrt{\epsilon} \vec{k} \cdot \vec{\nabla}_{\epsilon}-i \epsilon \nabla_{\epsilon}^{2}}{1+\epsilon}\right)^{-1}
$$

with

$$
\Delta=\theta+\frac{k^{2}}{1+\epsilon} .
$$

Correspondingly we use the operator $\mathcal{Q}^{\prime}$, defined as

$$
\mathcal{Q}^{\prime}=D \mathcal{L}^{\prime}-1-i \Delta-\frac{2 \sqrt{\epsilon} \vec{k} \cdot \vec{\nabla}_{\epsilon}-i \epsilon \nabla_{\epsilon}^{2}}{1+\epsilon},
$$

and observe that at the zeroth order in $\epsilon$

$$
\mathcal{Q}^{\prime}=\frac{D-1-\Delta^{2}}{1-i \Delta} \text {. }
$$

Hence, the correct scaling for the $D$ variable is

$$
D=1+\Delta^{2}+\sqrt{\epsilon} W .
$$

To determine $Z$ we need to know only the lower-order terms in the power expansions of $\mathcal{L}^{\prime}$ and $\mathcal{Q}^{\prime}$, which are

$$
\begin{gathered}
\mathcal{L}^{\prime}=\frac{1}{1-i \Delta}+\sqrt{\epsilon} \frac{2 \vec{k} \cdot \vec{\nabla}_{\epsilon}}{(1-i \Delta)^{2}}, \\
\mathcal{Q}^{\prime}=\sqrt{\epsilon} \frac{W+4 i \Delta \vec{k} \cdot \vec{\nabla}_{\epsilon}}{1-i \Delta}+2 \epsilon\left[\frac{W \vec{k} \cdot \vec{\nabla}_{\epsilon}}{(1-i \Delta)^{2}}+2 k^{2} \frac{1+i \Delta}{(1-i \Delta)^{2}} \nabla_{\epsilon}^{2}\right. \\
\left.+\frac{\Delta \nabla_{\epsilon}^{2}}{1-i \Delta}\right] .
\end{gathered}
$$

Using the above approximations we can write $Z=e^{i \vec{k} \cdot \vec{x}} Z^{\prime}$, with $Z^{\prime}$ given by

$$
\begin{aligned}
Z^{\prime}= & -\epsilon^{3 / 2} \frac{1+i \Delta}{(1-i \Delta)^{2}}\left(W+4 i \Delta \vec{k} \cdot \vec{\nabla}_{\epsilon}\right) F+\frac{\epsilon^{2}}{(1-i \Delta)^{3}}\{\sigma(1-i \Delta) \\
& \times\left(1+\Delta^{2}-\mu+|F|^{2}\right)-W^{2}-2 \Delta\left(1+\Delta^{2}\right) \nabla_{\epsilon}^{2}-2 W(3 \\
& +5 i \Delta) \vec{k} \cdot \vec{\nabla}_{\epsilon}-4(1+i \Delta)\left[(1+i \Delta) k^{2} \nabla_{\epsilon}^{2}+4 i \Delta\left(\vec{k} \cdot \vec{\nabla}_{\epsilon}\right)^{2}\right. \\
& \left.\left.+\vec{k} \cdot\left(\vec{\nabla}_{\epsilon} W\right)\right]\right\} F .
\end{aligned}
$$

The dynamical equations for $F$ and $W$ are then

$$
\begin{gathered}
\partial_{\tau} F=-\frac{2 \vec{k} \cdot \vec{\nabla}_{\epsilon}-i \sqrt{\epsilon} \nabla_{\epsilon}^{2}}{\epsilon(1+\epsilon)} F+\frac{\mathcal{Q}^{\prime} F+Z^{\prime}}{\sqrt{\epsilon}}, \\
\partial_{\tau} W=-\sigma\left\{\left(1+\Delta^{2}+\sqrt{\epsilon} W\right)\left[1+\operatorname{Re}\left(F^{*} \mathcal{L}^{\prime} F\right)\right]-\mu+\operatorname{Re}\left(F^{*} Z^{\prime}\right)\right\} .
\end{gathered}
$$

In Eq. (32b) one has to consider only the terms up to the order $\epsilon^{3 / 2}$ in $Z^{\prime}$. Equation (32) contains the long-term dynamics of solid-state lasers for any value of $\theta$. In this respect, they represent the solution of the single reduced model for stiff laser equations left open in Ref. [1]. For numerical purposes it may be convenient to expand the operators $\mathcal{L}^{\prime}$ and $\mathcal{Q}^{\prime}$ in power series. The general equations are quite convoluted and we do not report them here. In Secs. III B 1 and III B 2, however, we consider the special cases of positive detuning $\theta$ with plane-wave solutions and that of negative $\theta$ with $\Delta=0$ since the final equations can provide interesting insights in the spatiotemporal dynamics of solid-state lasers.

\section{Positive $\theta$}

For positive $\theta$ we only consider the case in which the stationary solution with the minimum threshold is the plane wave, $\vec{k}=0$. In this case the slowly varying amplitude $F$ coincides with the electric field $E$, and $\Delta=\theta$. Making a series expansion of $\mathcal{L}^{\prime}$ and $\mathcal{Q}^{\prime}$ we can write the dynamical equations explicitly in the following form:

$$
\begin{aligned}
\partial_{\tau} E= & \left\{\frac{i \nabla_{\epsilon}^{2}}{\sqrt{\epsilon}(1+\epsilon)}+\frac{W}{1-i \theta+\epsilon(1+i \theta)}+\frac{2 \sqrt{\epsilon} \theta}{1-i \theta+2 \epsilon} \nabla_{\epsilon}^{2}\right. \\
& -\frac{\epsilon W}{(1-i \theta)^{2}} i \nabla_{\epsilon}^{2}-\frac{\epsilon^{3 / 2}}{(1-i \theta)^{2}}\left[\frac{W^{2}}{1-i \theta}+(1+i \theta) \nabla_{\epsilon}^{4}\right. \\
& \left.\left.-\sigma\left(1+\theta^{2}-\mu+|E|^{2}\right)\right]\right\} E, \\
\partial_{\tau} W= & -\left\{\left(1+\theta^{2}+\sqrt{\epsilon} W\right)\left(1+\frac{|E|^{2}}{1+\theta^{2}}-\epsilon \operatorname{Re}\left[\frac{i E^{*} \nabla_{\epsilon}^{2} E}{(1-i \theta)^{2}}\right]\right)\right. \\
- & \left.\mu-\epsilon^{3 / 2} \frac{1-3 \theta^{2}}{\left(1+\theta^{2}\right)^{2}} W|E|^{2}\right\} .
\end{aligned}
$$

For small detunings, $\theta=\epsilon \delta$, these equations merge with Eq. (20) if the substitution $W \rightarrow W-\epsilon^{3 / 2} \delta^{2}$ is made to account for the fact that $W$ is defined in a different way in Eqs. (18) and (28).

From a numerical point of view, the first terms in the rhs of Eqs. (20a), (32a), and (33a) may be seen as a source of 
concern because the denominators contain the smallness parameter $\epsilon$. This, however, should not be the case since spatial scale renormalizations of the form $X=\epsilon^{3 / 4} x$ for Eqs. (20) and (33) and $X=\epsilon^{3 / 2} x$ for Eq. (32), respectively, provide an instant remedy. By applying these renormalizations one may consider the removal of several higher-order gradient and Laplacian terms from the reduced equations. These terms, however, may become significant at long spatial scales (i.e., small wave vectors) and should not be neglected arbitrarily. We note that the presence of separate spatial scales in the reduced equations from $\epsilon^{3 / 2} x$ to $\epsilon^{1 / 4} x$ is in any case an improvement with respect to the full equations where simultaneous accuracy over spatial scales from $\epsilon^{2} x$ to $x$ was required. For example, for typical value of $\epsilon$ of 0.025 , as considered in Sec. V, there is a net reduction in the range of relevant spatial scales of at least a factor of 3 .

The reduced equations contain all the long-term spatiotemporal dynamics of interest and evolve on time scales much slower than the original MB equations. For typical values of the decay rates for solid-state lasers, the time scale $\tau=\epsilon^{3 / 2} t$ is around 1000 times slower than that of the original model. This means that numerical simulations of the reduced equations performed on the $\tau$ scale can, in principle, attain long-term solutions 1000 times faster than those performed with the original equation (3). This point will be discussed further in Sec. V where the reduced equations have been numerically integrated and compared with the full original MB model.

\section{Negative $\theta$}

For negative detunings $\theta<0$ Eq. (32) explicitly depends on the wave vector of the traveling-wave solution that becomes stable above threshold. In order to show the relevance of these equations in this limit, we focus on the most unstable wave vector above lasing threshold that is given by

$$
\Delta=0, \quad k^{2}=-(1+\epsilon) \theta .
$$

Similar considerations are, however, valid for other wave vectors and even for spectral intervals of size $\epsilon$ around the most unstable wave vector. In the case of $\Delta=0$, Eq. (32) greatly simplifies since $Z^{\prime}$ reduces to

$$
\begin{aligned}
Z^{\prime}= & -\epsilon^{3 / 2} W F+\epsilon^{2}\left[\sigma\left(1-\mu+|F|^{2}\right)-W^{2}-6 W \vec{k} \cdot \vec{\nabla}_{\epsilon}-4 k^{2} \nabla_{\epsilon}^{2}\right. \\
& \left.-4\left(\vec{k} \cdot \vec{\nabla}_{\epsilon} W\right)\right] F .
\end{aligned}
$$

By retaining terms up to $\epsilon^{1 / 2}$ in Eq. (32), it can be further simplified to

$$
\partial_{\tau} F=-\frac{2 \vec{k} \cdot \vec{\nabla}_{\epsilon}-i \sqrt{\epsilon} \nabla_{\epsilon}^{2}}{\epsilon} F+W F+2 \sqrt{\epsilon}\left(W \vec{k} \cdot \vec{\nabla}_{\epsilon}+2 k^{2} \nabla_{\epsilon}^{2}\right) F,
$$

$$
\partial_{\tau} W=-\sigma\left[(1+\sqrt{\epsilon} W)\left(1+|F|^{2}\right)+2 \sqrt{\epsilon} \operatorname{Re}\left(F^{*} \vec{k} \cdot \vec{\nabla}_{\epsilon} F\right)-\mu\right] .
$$

These compact equations accurately describe the stability of the traveling-wave solution $\Delta=0$ for generic values of the pump parameter $\mu$. In the case of large negative detunings
Eq. (36) may also provide analytical results since they can be easily transferred to the Fourier space. These calculations are beyond the scope of this paper and will be presented elsewhere.

\section{DERIVATION OF THE REDUCED EQUATIONS: THE VCSEL CASE}

Following a procedure similar to that of Sec. III, we can also remove stiffness and reduce the number of equations in the VCSEL model considered in Ref. [13],

$$
\begin{gathered}
\partial_{t} E=i \nabla^{2} E+\epsilon[P-(1-i \alpha) E], \\
\partial_{t} P=\xi(D)(1-i \alpha) D E-[\xi(D)-i \epsilon \alpha] P, \\
\partial_{t} D=-\sigma \epsilon^{2}\left[D-\mu+\operatorname{Re}\left(E^{*} P\right)-d \nabla^{2} D\right]
\end{gathered}
$$

with

$$
\begin{gathered}
\xi(D)=\Gamma(D)(1-i \alpha)+2 i \delta(D), \\
\Gamma(D)=-0.07+2.80 D, \\
\delta(D)=\epsilon(\Delta+\alpha)+1.51(D-1) .
\end{gathered}
$$

Here $\alpha$ is the linewidth enhancement factor, $d$ is the diffusion coefficient of carriers, and $\Delta$ plays the role of an effective detuning parameter. With respect to [13] we have used a frequency shift $\epsilon \alpha$ to make the analysis more similar to that of the solid-state case.

Equation (37) describes the gain and dispersion mechanisms typical of semiconductor media through the modified relaxation rate of the polarization [13]. Similarly to the solidstate case, they admit traveling-wave solutions of the form

$$
\begin{gathered}
E=A \exp [i(\vec{k} \cdot \vec{x}-\omega t)], \\
P=\frac{(1-i \alpha) \xi(D) D E}{\xi(D)-i(\omega+\epsilon \alpha)}, \\
D=\mu_{\mathrm{th}}
\end{gathered}
$$

with

$$
\begin{gathered}
A=\sqrt{\mu-\mu_{\mathrm{th}}} e^{i \phi}, \\
\mu_{\mathrm{th}}=1+\frac{\left(k^{2}-\epsilon \Delta\right)^{2}-\epsilon^{2}(\Delta+\alpha)^{2}}{\Gamma(1)^{2}\left(1+\alpha^{2}\right)}+O\left(\epsilon^{3}\right), \\
\omega=k^{2}+O\left(\epsilon^{2}\right) .
\end{gathered}
$$

The above equations show that the parameter $\Delta$ plays a role analogous to that of $-\theta$ for the solid-state laser equations: if $\Delta>0$ the solution with minimum threshold is the traveling wave with $k^{2}=\epsilon \Delta$; if $\Delta<0$ it is the homogeneous solution $k=0$.

To proceed with the derivation of the $\mathrm{CM}$ associated with Eq. (37) we observe that Eq. (39b) can be written as

$$
P=(1-i \alpha) D \mathcal{L} E
$$

with the operator $\mathcal{L}$ given by 


$$
\mathcal{L}=\left[1+i \xi(D)^{-1}\left(\nabla^{2}-\epsilon \alpha\right)\right]^{-1} .
$$

If we then use the dynamical variable $Z$ and the space-time operator $\mathcal{D}$ defined as

$$
\begin{aligned}
(1-i \alpha) Z & =P-(1-i \alpha) D \mathcal{L} E, \\
\mathcal{D} & =\partial_{t}-i \nabla^{2}
\end{aligned}
$$

we can recast Eq. (37) in a form similar to Eq. (11),

$$
\begin{gathered}
\mathcal{D} Z=-\xi \mathcal{L}^{-1} Z-\partial_{t} D(\mathcal{L} E)-D \mathcal{L}(\mathcal{D} E), \\
\mathcal{D} E=\epsilon(1-i \alpha)(\mathcal{Q} E+Z),
\end{gathered}
$$

$$
\begin{aligned}
\mathcal{D} D= & -\sigma \epsilon^{2}\left\{D-\mu+D \operatorname{Re}\left[(1-i \alpha) E^{*} \mathcal{L} E\right]+\operatorname{Re}\left[(1-i \alpha) E^{*} Z\right]\right. \\
& \left.-d \nabla^{2} D\right\}-i \epsilon \nabla_{\epsilon}^{2} D
\end{aligned}
$$

with $\mathcal{Q}=D \mathcal{L}-1$. These equations admit a center manifold. As before, we can expand the fast variable $Z$ in a power series of the smallness parameter $\epsilon$. At second order in $\epsilon$ one obtains $Z=\epsilon Z_{1}+\epsilon^{2} Z_{2}$ with

$$
\begin{gathered}
Z_{1}=-(1-i \alpha) \mathcal{L}\left[\xi^{-1} D \mathcal{L}(\mathcal{Q} E)\right], \\
Z_{2}=\sigma \mathcal{L}\left\{\xi^{-1}\left\{D-\mu+D \operatorname{Re}\left[(1-i \alpha) E^{*} \mathcal{L} E\right]\right\}(\mathcal{L} E)\right\} \\
+(1-i \alpha)^{2} \mathcal{L}\left\{\xi^{-1} D \mathcal{L}^{2}\left[\xi^{-1} D \mathcal{L}(\mathcal{Q} E)\right]\right\} \\
+(1-i \alpha)^{2} \mathcal{L}\left\{\xi^{-1} \mathcal{L}\left[D \mathcal{L}\left(\mathcal{Q}^{2} E\right)\right]\right\} \\
+i \mathcal{L}\left(\xi^{-1} \frac{\partial Z_{1}}{\partial D} \nabla_{\epsilon}^{2} D\right)
\end{gathered}
$$

At this point, in the solid-state laser case, we analyzed the limits of small and large detunings separately. In the VCSEL case, however, the assumptions made to derive model (37) restrict their validity to a frequency range of the order $\epsilon$ around the frequency of maximum gain [13]. Consistently, we assume that the effective detuning $\Delta$ is at most of order 1 , which means that we consider the limit of small detuning, where the traveling-wave solutions are characterized by long spatial scales and slow temporal scales. Hence, we replace $\nabla^{2}$ with $\epsilon \nabla_{\epsilon}^{2}$ and write the reduced equations for the VCSEL as

$$
\begin{gathered}
\partial_{\epsilon t} E=i \nabla_{\epsilon}^{2} E+(1-i \alpha)(\mathcal{Q} E+Z), \\
\partial_{\epsilon t} D=-\sigma \epsilon\left\{D-\mu+D \operatorname{Re}\left[(1-i \alpha) E^{*} \mathcal{L} E\right]+\operatorname{Re}\left[(1-i \alpha) E^{*} Z\right]\right. \\
\left.-d \epsilon \nabla_{\epsilon}^{2} D\right\} .
\end{gathered}
$$

These equations are similar to Eq. (17) obtained for solidstate lasers in the same limit of small detuning. It is then possible to proceed as in Sec. III A observing that, as before, the operator $\mathcal{Q}$ admits the singular expansion of the carrier density variable $D=1+\sqrt{\epsilon} W$. As a consequence the function $\xi(D)[$ now $\xi(W)]$ contains terms of different order in $\epsilon$

$$
\begin{gathered}
\xi(W)=\xi_{0}+\xi_{1} \sqrt{\epsilon} W+2 i \epsilon(\Delta+\alpha), \\
\xi_{0}=\Gamma(1)(1-i \alpha),
\end{gathered}
$$

$$
\xi_{1}=2.80+i(3.02-2.80 \alpha) .
$$

With the above substitutions, and with a series expansion of the operators $\mathcal{L}$ and $\mathcal{Q}$, the VCSEL equations read

$$
\begin{aligned}
\partial_{\tau} E= & \left\{\frac{i \nabla_{\epsilon}^{2}}{\sqrt{\epsilon}}+(1-i \alpha) W+\epsilon^{3 / 2} \frac{(\Delta+\alpha)^{2}-\left(\nabla_{\epsilon}^{2}+\Delta\right)^{2}}{\Gamma(1)^{2}\left(1+\alpha^{2}\right)}(1+i \alpha)\right. \\
& -i \frac{\sqrt{\epsilon}}{\Gamma(1)}\left[\frac{\xi_{0}(1+\sqrt{\epsilon} W)}{\xi_{0}+\xi_{1} \sqrt{\epsilon} W}-\frac{\epsilon}{\Gamma(1)}\right]\left(\nabla_{\epsilon}^{2}-\alpha\right)-(1-i \alpha) \\
& \times \frac{\epsilon W}{\Gamma(1)}\left[1+\left(1-\frac{\xi_{1}}{\xi_{0}}\right) \sqrt{\epsilon} W\right]+\frac{\epsilon^{3 / 2} \sigma}{\Gamma(1)} \\
& \left.\times\left(1-\mu+|E|^{2}\right)\right\} E, \\
\partial_{\tau} W= & -\sigma\left\{(1+\sqrt{\epsilon} W)\left[1+|E|^{2}-\frac{\epsilon \operatorname{Re}\left(i E^{*} \nabla_{\epsilon}^{2} E\right)}{\Gamma(1)}\right]-\mu\right. \\
& +\frac{\epsilon^{3 / 2} W}{\Gamma(1)} \operatorname{Re}\left[i \frac{\xi_{1}}{\xi_{0}} E^{*}\left(\nabla_{\epsilon}^{2}-\alpha\right) E\right]-\frac{\epsilon^{3 / 2} W}{\Gamma(1)}|E|^{2} \\
& \left.-d \epsilon^{3 / 2} \nabla_{\epsilon}^{2} W\right\} .
\end{aligned}
$$

These equations evolve on a time scale much slower that the original VCSEL equations since the time scale $\tau=\epsilon^{3 / 2} t$ is around 1000 times slower than that of the original model for typical values of $\epsilon$ on the order of $10^{-2}$. In Sec. V we present numerical simulations where the reduced equation (49) is compared with the full model (37).

Similarly to the solid-state laser model, we can consider the close-to-threshold limit of Eq. (49) which, after a redefinition of the reference frequency, reads

$$
\begin{gathered}
\partial_{\tau} E=\frac{i \nabla_{\epsilon}^{2} E}{\sqrt{\epsilon}[1+\epsilon / \Gamma(1)]}+(1-i \alpha) W E+\frac{\epsilon^{3 / 2}}{\Gamma(1)^{2}}\left(\frac{(\Delta+\alpha)^{2}}{\left(1+\alpha^{2}\right)}\right. \\
\left.-\frac{\left(\Delta+\nabla_{\epsilon}^{2}\right)^{2}}{(1-i \alpha)}\right) E \\
\partial_{\tau} W=-\sigma\left(1+\sqrt{\epsilon} W+|E|^{2}-\mu\right) .
\end{gathered}
$$

In Appendix $\mathrm{C}$ we discuss similarities and differences between these equations and the analogous ones derived by Mercier and Moloney [3]. The differences are due to the fact that, unlike in the two-level case where the starting equations are always the well-established Maxwell-Bloch equations, in the semiconductor laser case various sets of effective Maxwell-Bloch equations have been proposed, and those used in [3] differ from ours in some basic assumptions which are made on the complex semiconductor susceptibility [13].

\section{ANALYTICAL AND NUMERICAL COMPARISONS BETWEEN FULL AND REDUCED MODELS}

In this section we provide evidence that the reduced models obtained in Secs. II-IV provide a faithful representation of the long-term dynamics of the full solid-state laser and 
VCSEL models. Since the equations and, in particular, the dynamical behavior of the solid-state laser are simpler than those of the VCSEL, we start from these models, where the stability of the steady states can be assessed analytically.

\section{A. Solid-state laser models}

We first checked the validity of the reduced model by comparing the results of its stability analysis with those of the full Maxwell-Bloch model. Although the degree of the characteristic polynomial is 5 for the full model and 3 for the reduced one, two eigenvalues of the full model always have a large negative real part $[2,10]$, and so the comparison is made using its remaining three eigenvalues.

We considered the most general set of reduced equation (32) which is valid for any value of $\theta$ and $\vec{k}$. For any $\vec{k}$ these equations admit the exact stationary solution

$$
F=\sqrt{\mu-1-\Delta^{2}} e^{i \phi}, \quad W=0,
$$

which, taking into account the change in variable (22), coincides with the traveling-wave solution of the full model, given by Eqs. (5) and (6). In fact, for $F$ spatially homogeneous and $W=0$, we have $Z^{\prime}=0$ and Eq. (32) reduces to Eq. (14) which admit the traveling waves as exact solutions. However, as mentioned above, the inclusion of the term $Z^{\prime}$ in the reduced model provides a better agreement with the stability and dynamics of the full model. We also compared the eigenvalues governing the stability of the traveling-wave solution of the full and reduced models with those of the CSH model of $[2,10]$.

\section{1. $|\theta|=O(\epsilon)$}

To compare the eigenvalues of a perturbation of wave vector $\vec{q}$ of the CSH model with those of the full and reduced models, we first considered the same set of parameters used in [10]. As in that paper, we limit our analysis to the onedimensional case, so that the wave vectors $\vec{k}$ of the stationary traveling wave and $\vec{q}$ of the perturbation are either parallel or antiparallel and they can be treated as real numbers. In other words, we neglect the additional degree of freedom represented by the angle between $\vec{k}$ and $\vec{q}$.

Figure 2(a) corresponds exactly to Fig. 7 of [10], with the solid lines for the full Maxwell-Bloch equations, the dotted lines for the CSH model, and the dashed lines for our reduced model (32). Note that the scale of the horizontal axis differs by a factor of 10 from that of [10] because there the Laplacian is multiplied by a factor $a=0.01$ which we set equal to 1 in our equations. For the same reason the wave vector of the stationary solution is $k=0.09$ here and $k=0.9$ in [10]. In Fig. 2(b), which is an enlargement of Fig. 2(a), we also show the eigenvalues obtained with the reduced model and $Z^{\prime}=0$ (dashed-dotted lines).

From this figure we can draw the following conclusions:

(i) for small $q$, the reduced model agrees extremely well with the full model;

(ii) for any $q$, the reduced model reproduces the behavior of the neutral eigenvalue, the one whose real part is zero for $q=0$ and tends to a constant value for large $q$, much better than the CSH model; and

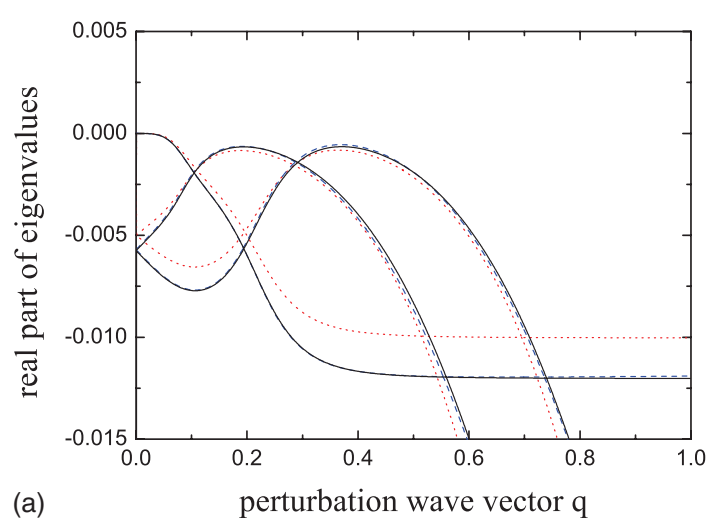

(a)

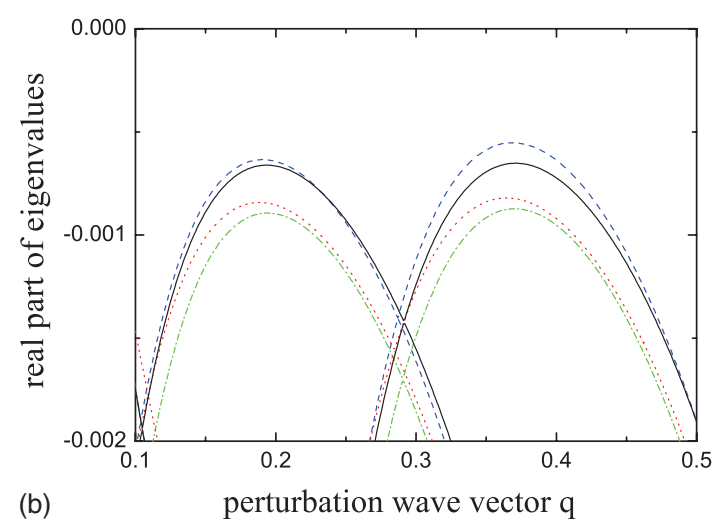

FIG. 2. (Color online) Small and negative $\theta . \epsilon=0.1, \sigma=1.0$, $\theta(1+\epsilon)=-0.001$, and $\mu=1.2$. Stability of the traveling-wave solution with wave vector $k=0.09$. In (a) the three eigenvalues with largest real part of the full equations [solid lines (black online)] are compared with the eigenvalues of the $\mathrm{CSH}$ model [dotted lines (red online)] and of the reduced equations [dashed lines (blue online)]. In (b), which is an enlargement of (a), the dashed-dotted lines (green online) show the eigenvalues calculated with the reduced model and $Z^{\prime}=0$.

(iii) for the other two eigenvalues, both displaying a maximum, we observe that the maximum values are calculated more precisely with the reduced model than with the $\mathrm{CSH}$ model. However, the reduced model with $Z^{\prime}=0$ has a similar degree of accuracy as the CSH model.

\section{Negative $\theta$ and $|\theta|=O(1)$}

In Fig. 3 we considered a much larger value of $\theta$, namely, $\theta(1+\epsilon)=-1$, for which the equations admit solutions that are rapidly oscillating traveling waves. For this value of $\theta$, the traveling wave with minimum threshold corresponds to the wave vector $k=1$. For generality purposes, we analyzed the stability of the traveling wave with wave vector $k=0.97$. We observe first that even for such a large value of $\theta$, and also of the pump parameter $\mu=3$, the CSH model still approximates the full model quite well, at least for the relevant eigenvalues, i.e., those whose real part has positive values. However, our reduced model performs even better and its results are indistinguishable from those of the full model, even in the enlargement shown in Fig. 3(b). Figure 3(b) also shows that, as before, the reduced model with $Z^{\prime}=0$ has a similar degree 

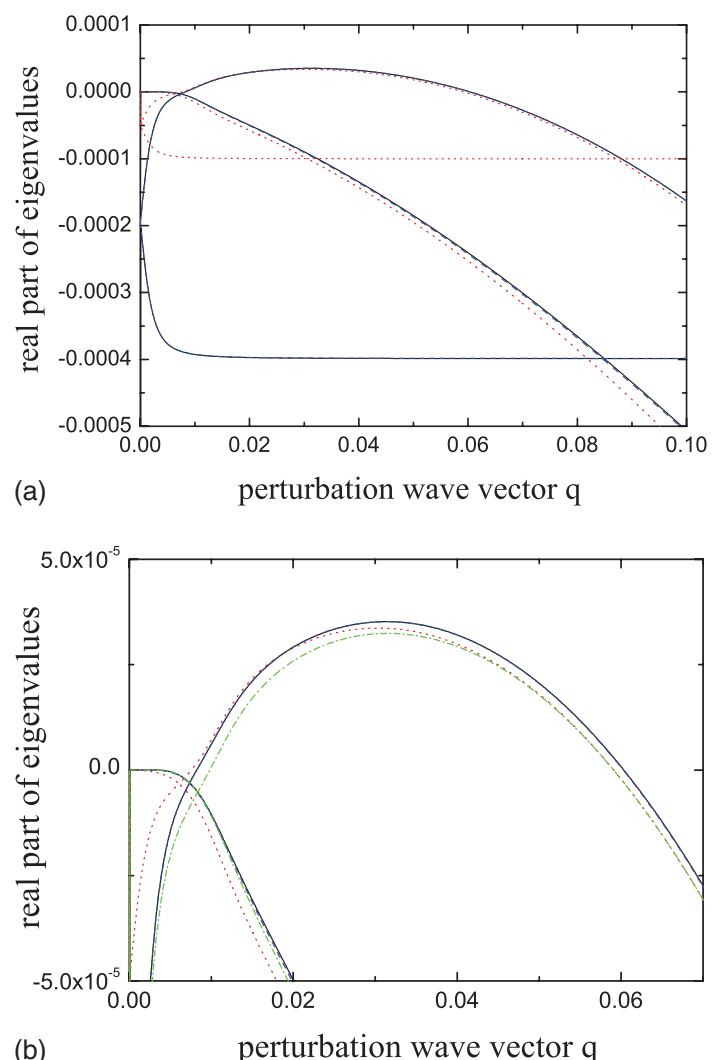

FIG. 3. (Color online) Large and negative $\theta . \epsilon=0.01, \sigma=1.0$, $\theta(1+\epsilon)=-1$, and $\mu=3$. Stability of the traveling-wave solution with wave vector $k=0.97$. The meaning of the different line styles is the same as in Fig. 2.

of accuracy as the CSH model, thus emphasizing the beneficial role played by $Z^{\prime}$ in our equations.

\section{Positive $\theta$ and $|\theta|=O(1)$}

For positive values of $\theta$ the stable stationary solution at laser threshold is the homogeneous one. We analyzed its stability for the following set of parameters: $\epsilon=0.025, \sigma=0.16$ $\left(\sigma \epsilon^{2}=10^{-4}\right), \theta(1+\epsilon)=0.1$, and $\mu=1.3$. The value of $\theta$ is large in the sense that it is about 4 times larger than $\epsilon$, although it is still smaller than 1 . In this case, since we are analyzing the stability of the homogeneous solution with null wave vector, the analysis extends beyond the one-dimensional limit and $q$ can be regarded as the modulus of a wave vector $\vec{q}$ with arbitrary direction in the transverse plane.

Figure 4 shows that with our choice of parameters the reduced model again agrees very well with the full model, while the CSH model provides quite inaccurate results, even though these parameters are not too far from the limit of validity of the CSH equations. In particular, the CSH model does not catch the instability that is present in the full model in a small interval centered around $q \simeq 0.047$. Figure 4(b), however, shows that this instability is described by the reduced model with almost the same accuracy as the full model. To be precise, the instability threshold is $\mu=1.2896$ for the full model and $\mu=1.2905$ for the reduced model. In contrast with the previous cases, for these parameters the
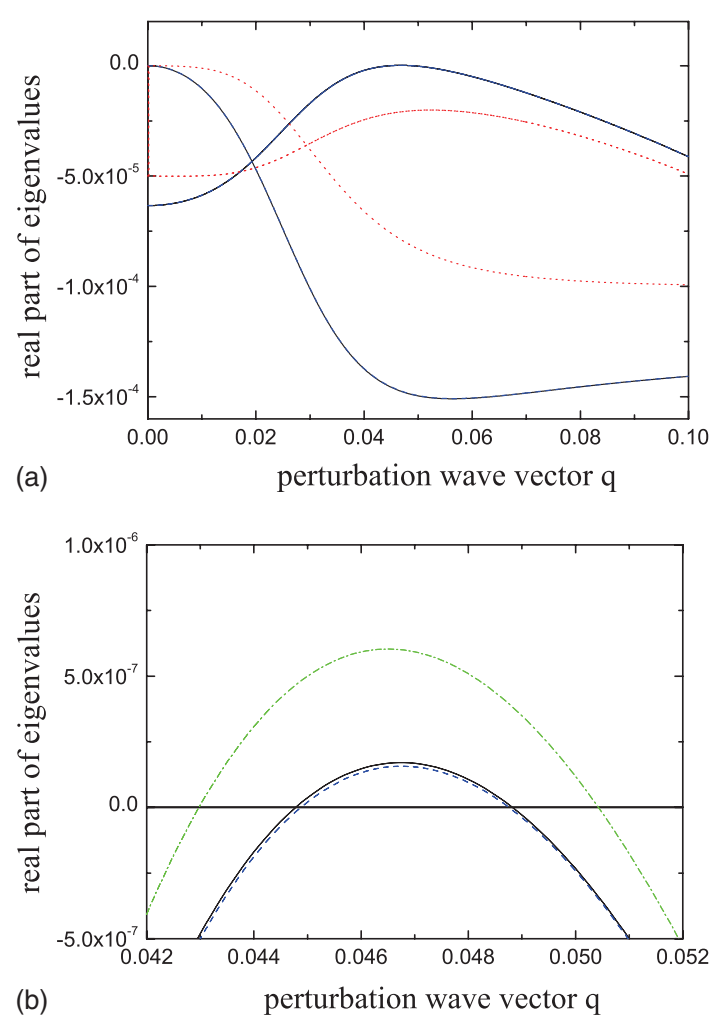

FIG. 4. (Color online) Positive $\theta . \epsilon=0.025, \sigma=0.16, \theta(1+\epsilon)$ $=0.1$, and $\mu=1.3$. Stability of the homogeneous solution $(k=0)$. The meaning of the different line styles is the same as in Figs. 2 and 3.

reduced model with $Z^{\prime}=0$ is far more precise than the $\mathrm{CSH}$ model, although it predicts an instability threshold at $\mu$ $=1.2645$.

To confirm that the reduced equations are indeed capable of describing the instabilities of the full equations, we numerically integrated with an accurate split-step algorithm both full and reduced equations with these parameters. In both cases the pump $\mu$ was first gradually increased from 1.05 to 1.45 and then decreased from 1.45 back to 1.05 . For each value of the pump the equations were integrated for a total time of $2.5 \times 10^{6}$ in the units of the full model (3) with the final values of the variables used as the initial conditions of the next pump step.

From the calculation of the stability eigenvalues [see Fig. 4(b)] we expect the instability threshold for both full and reduced models to be around $\mu \approx 1.29$. Figure 5 shows that the simulations are affected by critical slowing down in the vicinity of the bifurcation point. This is expected from the calculation of the stability eigenvalues since the magnitude of the real part of the eigenvalue is extremely small above threshold. To avoid this problem, one needs to integrate the equations for a much longer period of time. We have therefore increased the simulation time of the reduced equations by a factor of 10 both increasing and decreasing the pump $\mu$ as before. We have obtained a reduction in the interval of the instability thresholds between the forward and reverse simulations of a factor of 3 with a final positioning of the instability threshold at $1.291 \pm 0.002$.

One would expect to see similar results for the full equations, but a simulation of the full equations of the necessary 


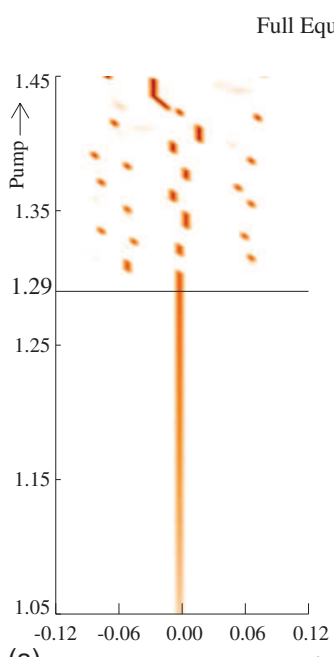

ll Equations

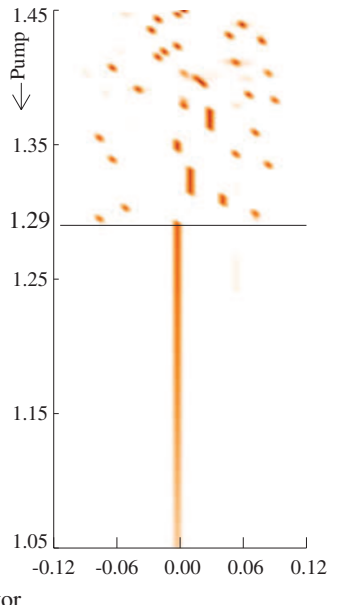

Reduced Equations

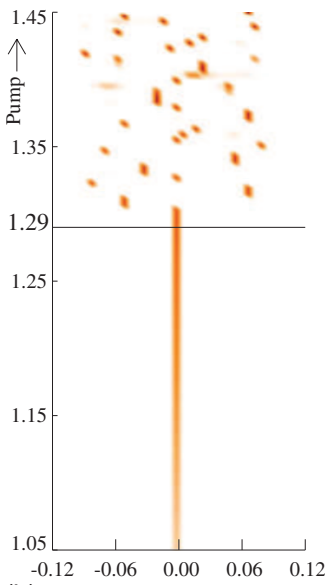

(b)

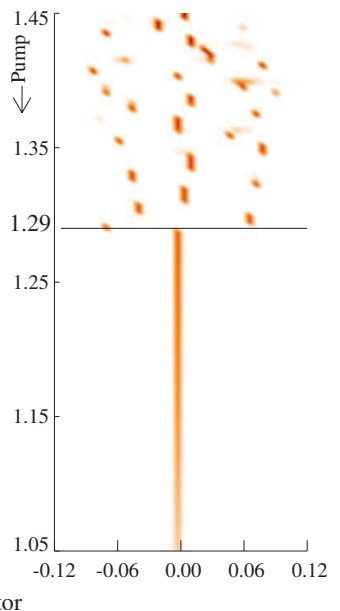

k-vector

FIG. 5. (Color online) Far-field (wave-vector space) components of the numerical integration of the (a) full model (3) and (b) reduced model (33) for the solid-state laser case. In the left panels of (a) and (b), the pump $\mu$ is gradually increased from 1.05 to 1.45 , while in the right panels it is decreased from 1.45 to 1.05 . Parameters are $\epsilon=0.025, \sigma=0.16$, and $\theta=0.1$.

duration is simply not feasible on our computer resources. Note that numerical integration of the reduced equations is done on a time scale given by $\tau=\epsilon \sqrt{\epsilon} t$ which is around 1000 times slower than that of the original model for typical values $\epsilon \approx 10^{-2}$ of the field decay rate of solid-state lasers. This means that numerical simulations of Eq. (33) performed on the $\tau$ scale can possibly attain long-term solutions around 1000 times faster than those performed on the original equation (3). In the above simulation $\epsilon=0.025$ which gives a time scale of $\tau=253 t$. However, since in this case $\sigma<1$, it is convenient to include it into the singular perturbation expansion, so that we obtain $\tau=633 t$. In practice, the larger number of numerical steps required in the reduced equations meant that for the case considered here simulations of the reduced equations were performed 285 times faster than simulations of the full equations. This is still a very significant speedup: simulations that would take around 1 day using the reduced model would require almost 1 year using the full model. Clearly this reduction in CPU time will have a huge impact on the range of numerical investigations which are now made possible.

From Fig. 5 and the above considerations, it is clear that the instability of the homogeneous solution is well reproduced by the reduced models and is missed by the CSH. Above this instability (a Hopf bifurcation at a finite critical wave vector $q_{c}$ ) irregular spatiotemporal oscillations are observed. In order to demonstrate that the agreement between full and reduced models extends way above the homogeneous state instability, we present in Fig. 6 a comparison of their final output intensities for a wide range of pump values. The agreement is excellent since the small difference between the solid (full) and dashed (reduced) lines above the instability threshold is due to the different phase of the irregular oscillation at the end of each simulation. These results demonstrate not only the accuracy of the reduced equations in finding important laser instabilities of the original models, but also their usefulness in laser modeling since the large CPU gain factor makes otherwise unfeasible simulations possible.

\section{B. VCSEL models}

The comparison of the full and reduced VCSEL models was based on the analysis of the long-wavelength instability of the homogeneous solution which is predicted by Eq. (37) [known as Lugiato-Oldano-Narducci (LON) instability]. In [13] it was shown that at the leading order in $\epsilon$ the homogeneous solution is unstable against any wave vector of modulus $k$ with

$$
k^{2}<2 \epsilon \alpha \frac{\mu-1}{\mu} .
$$

The unstable wave vectors are limited to the band $0<k^{2}$ $<2 \epsilon \alpha$, and no critical wave vector for this instability exists. For any $\mu$ larger than 1 (the laser threshold at order 0 in $\epsilon$ ) there is always a band of unstable wave vectors. In an infinitely extended device this means that the homogeneous solution would be unstable just above threshold with respect to perturbations with infinite wavelength. In a real device, however, as well as in numerical simulations, the wave vectors

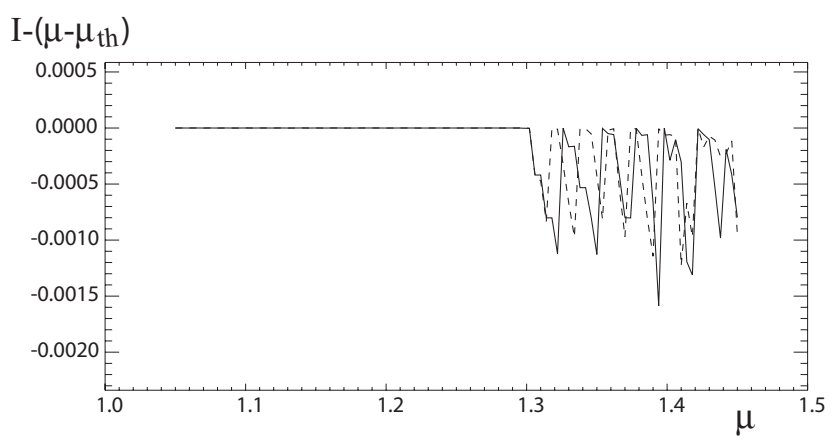

FIG. 6. Comparison of the difference between the laser intensity $I$ and that of the steady state $\left(\mu-\mu_{\mathrm{th}}\right) \quad(k=0)$ obtained from the numerical integration of the full model (3) (solid line) and reduced model (33) (dashed line) for the solid-state laser case. Parameters are the same as in Fig. 5. 


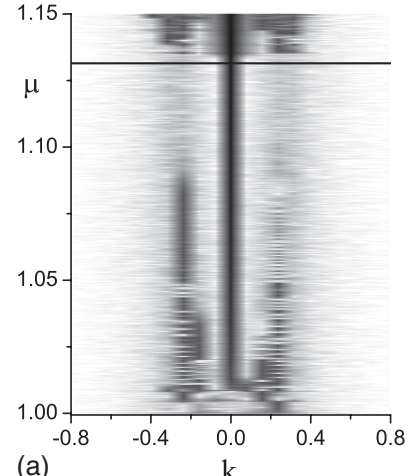

(a)

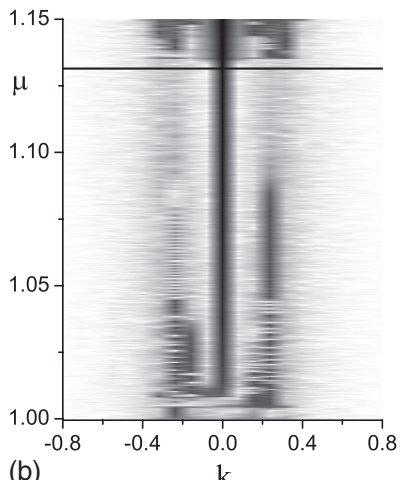

(b)

FIG. 7. Comparison between simulation results for VCSEL models showing the far-field spectrum versus the pump amplitude $\mu$ using the full (left panel) and reduced (right panel) models and $\epsilon=0.025, \sigma=0.16, \Delta=2$, and $\alpha=1.08$.

are discrete, and a minimum wave vector $k_{1}=2 \pi / L$ exists, where $L$ is the size of the device (or of the integration window). The instability threshold of the homogeneous solution is then given by

$$
\mu_{i}=\left(1-\frac{k_{1}^{2}}{2 \epsilon \alpha}\right)^{-1} .
$$

In [13] it was found that this instability manifests itself for both signs of the effective detuning $\Delta$. If $\Delta<0$, the laser selects the homogeneous solution just above threshold, and such solution remains stable up to $\mu_{i}$. If $\Delta>0$ the solution selected at threshold is one of the two traveling waves with wave vector closest to $\pm \sqrt{\epsilon \Delta}$. The numerical simulations, however, show that the traveling wave becomes unstable almost immediately above threshold and, upon increasing the pump $\mu$, a complex spatiotemporal dynamics appears until, for a value of $\mu$ smaller than $\mu_{i}$, the electric field jumps to the homogeneous solution $k=0$. For larger values of $\mu$ the behavior of the VCSEL with positive $\Delta$ is substantially identical to that observed for $\Delta<0$, where the homogeneous solution is stable until the value of $\mu_{i}$ is reached.

We have integrated the full and reduced VCSEL equations for a positive value of $\Delta$ because in this case the dynamics is richer and the comparison is more meaningful. With both models we have found the same sequence when increasing the pump from below $\mu_{\text {th }}$ to above $\mu_{i}$ : (i) traveling wave with $|k| \simeq \sqrt{\epsilon \Delta}$ at $\mu \simeq \mu_{\text {th }}$, (ii) competition among traveling waves with different wave vectors, (iii) coexistence of on-axis emission and a traveling wave with $|k| \simeq \sqrt{\epsilon \Delta}$, (iv) on-axis emission, (v) instability triggered by wave vectors $\pm k_{1}$ at $\mu \simeq \mu_{i}$, and (vi) complex dynamics. The agreement between the two models is very good even in the details, as shown in Fig. 7 .

The horizontal lines show the thresholds of the LON instability in the two models. According to the approximate formula (53), the threshold with our choice of parameters $\left(k_{1}^{2} / \epsilon=0.25\right.$ and $\left.\alpha=1.08\right)$ is $\mu_{i}=1.13089$. The integration of the dynamical equations reveals that the thresholds are actually slightly higher than this value: $\mu_{i}=1.13145$ for the full model and $\mu_{i}=1.13146$ for the reduced one. The LON in-

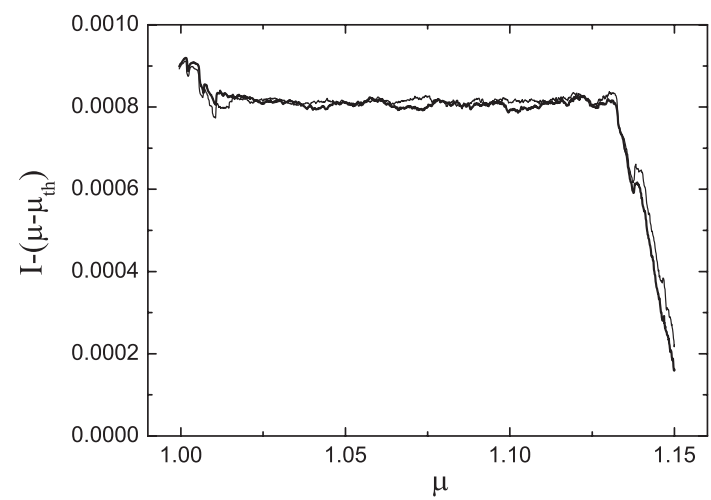

FIG. 8. Comparison of the difference between the laser intensity and that of the steady state $(k=0)$ obtained from the numerical integration of the full model [Eqs. (37) and (38)] (thick line) and reduced model (49) (thin line) for the VCSEL case. Parameters are the same as in Fig. 7.

stability is captured extremely well by the reduced model. The differences between the analytical and the numerical thresholds are due to terms of the order $\epsilon^{2}$ which were neglected in the derivation of Eq. (53). In the dynamical simulations shown in Fig. 7 the instability threshold is even larger because of the inertia of the system when a parameter (in this case $\mu$ ) varies in time. We chose the time step in such a way that the difference between the dynamic and the static thresholds was the same in both models. This can be accomplished when the time step in the full model is 248 times smaller than in the reduced model. Because of the large number of numerical calculations required to evaluate $Z$ the integration time is 146 less for the reduced model. This gain factor is still impressive since VCSEL simulations that may require several months of CPU time with the full equations can now be accurately performed within a single day with our reduced system of equations.

For completeness, we show in Fig. 8 a comparison of the final output intensities between the full and reduced VCSEL models. As in the case of solid-state lasers, the agreement is excellent and the small discrepancies between the thick (full) and thin (reduced) lines are due to the different phase of the irregular oscillations where the homogeneous state is unstable.

The integration of Eq. (50), which hold in the vicinity of the laser threshold, produces a dynamical behavior very similar to that shown in Fig. 7, with the only difference that the instability threshold $\mu_{i}$ is underestimated, since it is given by

$$
\mu_{i, \mathrm{ap}}=1+\frac{q_{1}^{2}}{2 \epsilon \alpha} .
$$

The larger the term $q_{1}^{2} /(2 \epsilon \alpha)$, the larger the difference between $\mu_{i}$ and $\mu_{i, \text { ap }}$. With our parameters we have $\mu_{i \text {,ap }}$ $=1.116$. Equation (54) holds also for the CSH model of Mercier and Moloney [3,13].

\section{CONCLUSIONS}

We have shown that center manifold techniques can be successfully applied to a variety of stiff laser models. We 
have first demonstrated the existence of the slow manifolds by recasting partial differential equations of solid-state and VCSEL lasers into forms appropriate for the applications of the mathematical theorems. We have then reduced the number of dynamical variables to those that are relevant to the long-term spatiotemporal evolution. Perturbative evaluations of the slow manifolds have been made possible by the large separation of time scales in the dynamics of the physical variables in laser devices.

The reduced models successfully describe laser dynamics over wide ranges of parameter space for both solid-state and semiconductor-based devices. In particular we have been able to remove the "close to threshold" and small detuning constraints that have affected previous attempts in the identification of the relevant laser variables. A large part of the scientific and commercial success of solid-state and VCSEL devices is related to their high gain and possible operation well above the lasing threshold. Our reduced models, although fairly complex for analytical manipulation, are very useful in numerical integration. We have shown that they correctly capture several spatiotemporal regimes of operation of the full equations, from traveling-wave dynamics to multitransverse modes locking and from oscillatory behavior at finite wave vectors to irregular motion. The bifurcation diagrams are reproduced with an extraordinary accuracy although the reduced models are obtained via a perturbation expansion. For example, we have shown in Sec. V A 3 that our equations reproduce accurately a Hopf bifurcation with a finite transverse wave vector which is missed in close-tothreshold models.

One of the main benefits of the use of the reduced models derived in this paper remains the large reduction in the $\mathrm{CPU}$ time of their numerical simulations with respect to the full set of equations. We have shown that even in the presence of relatively "large" $\epsilon$, the smallness parameter of the time scale separations, CPU gain factors of around 200 are routinely obtained in the numerical implementation of the reduced equations. For certain laser devices where $\epsilon$ is on the order of $10^{-3}$ instead of $10^{-2}$, CPU gain factors can easily be around $10^{4}$, thus making it feasible to simulate spatiotemporal effects in these devices. The analytical expressions of the slow manifolds derived here are beneficial to numerical simulations with either fixed or variable time steps.

Applications of the reduced models of solid-state and VCSEL equations are in both academic and industrial researches. Static and moving cavity solitons in these reduced models have already been identified and compared to their counterparts in the full set of equations. In particular, the application of cavity soliton lasers to all-optical delay lines [14] can have benefit greatly from faster and accurate numerical simulations.

\section{ACKNOWLEDGMENTS}

We acknowledge support from the EU network grant FunFACS. AMY acknowledges financial support from the National Endowment for Science, Technology and the Arts, and Strathclyde RDF. G.J.d.V. acknowledges support under Project No. FIS2005-07931-C03-01 funded by the Spanish
Ministerio de Educación y Ciencia and European Union FEDER.

\section{APPENDIX A}

In this appendix we show how Eq. (14) can be straightforwardly derived from the full Maxwell-Bloch equations. We start by noticing that Eq. (3b) admits the following solution:

$$
P=\left(1-i \theta+\partial_{t}\right)^{-1} D E .
$$

After expanding the linear operator and indicating with $\partial_{t}^{n}$ the $n$th time derivative, we have

$$
\begin{aligned}
P & =\frac{1}{1-i \theta} \sum_{n=0}^{\infty}\left(\frac{-\partial_{t}}{1-i \theta}\right)^{n} D E \\
& =\frac{1}{1-i \theta} \sum_{n=0}^{\infty}\left(\frac{-1}{1-i \theta}\right)^{n} \sum_{m=0}^{n} \frac{n ! \partial_{t}^{m} D \partial_{t}^{n-m} E}{m !(n-m) !} .
\end{aligned}
$$

In the sums it is convenient to separate the terms with $m$ $=0$ from the rest, so we can write

$$
\begin{aligned}
P= & \frac{1}{1-i \theta}\left[D \sum_{n=0}^{\infty}\left(\frac{-\partial_{t}}{1-i \theta}\right)^{n} E\right. \\
& \left.+\sum_{n=0}^{\infty}\left(\frac{-1}{1-i \theta}\right)^{n} \sum_{m=1}^{n} \frac{n ! \partial_{t}^{m} D \partial_{t}^{n-m} E}{m !(n-m) !}\right] .
\end{aligned}
$$

The last term is at least of the order $\epsilon^{2}$. As for the first term, we notice that Eq. (3a) can be rewritten as

$$
\partial_{t} E=\frac{i \nabla^{2} E}{1+\epsilon}+\epsilon\left[P-(1+i \theta) E+\frac{i \nabla^{2} E}{1+\epsilon}\right] .
$$

Hence in Eq. (A3) we can set $\partial_{t} E=i \nabla^{2} E /(1+\epsilon)+O(\epsilon)$ and obtain

$$
\begin{gathered}
P=\frac{D}{1-i \theta} \sum_{n=0}^{\infty}\left(\frac{-i \nabla^{2}}{(1+\epsilon)(1-i \theta)}\right)^{n} E+O(\epsilon) \\
=D\left(1-i \theta+\frac{i \nabla^{2}}{1+\epsilon}\right)^{-1} E+O(\epsilon)=D \mathcal{L} E+O(\epsilon) .
\end{gathered}
$$

Incidentally we note that this derivation bears some similarities with the one of Ref. [15] in the context of class A lasers.

\section{APPENDIX B}

In this appendix we want to show that, in the close-tothreshold limit, Eq. (21) is equivalent to the CSH model of [2], numbered in that paper as Eq. (5). We first rewrite Eq. (21) using the unscaled time $t$ and the substitutions $W \rightarrow(D$ $-1) / \sqrt{\epsilon}, \delta \rightarrow \theta / \epsilon$, and $\nabla_{\epsilon}^{2} \rightarrow \nabla^{2} / \epsilon$, obtaining

$$
\partial_{t} E=\frac{i \nabla^{2} E}{1+\epsilon}+\epsilon\left[D-1-\left(\theta-\nabla^{2}\right)^{2}\right] E,
$$




$$
\partial_{t} D=-\sigma \epsilon^{2}\left(D+|E|^{2}-\mu\right) .
$$

We then consider Eq. (5) of [2] and observe that, if we remove the oscillating term $-i \sigma \Omega \psi$ and apply the limit $\sigma, a, \Omega=O(\epsilon)$, they can be written as

$$
\begin{gathered}
\partial_{t} \psi=\frac{i a \nabla^{2} \psi}{1+\sigma}+\sigma\left[r-1-n-\left(\Omega+a \nabla^{2}\right)^{2}\right] \psi, \\
\partial_{t} n=-b n+|\psi|^{2} .
\end{gathered}
$$

The detuning $\Omega$ in [2] is measured with respect to the empty cavity frequency rather than the lasing frequency, as in our model. Moreover, it is defined with the opposite sign; hence, $\Omega=-\theta(1+\epsilon)$ in our notation. With this and the further substitutions $\sigma \rightarrow \epsilon, b \rightarrow \sigma \epsilon^{2}, r \rightarrow \mu, \psi \rightarrow \sqrt{b} E, n \rightarrow \mu-D, x / \sqrt{a}$ $\rightarrow x$, and $y / \sqrt{a} \rightarrow y$, Eq. (B1) is equivalent to Eq. (B2) up to fourth order in $\epsilon$, if $\theta=O(\epsilon)$.

\section{APPENDIX C}

In this appendix we compare the close-to-threshold limit of the VCSEL equations given by Eq. (50) with the CSH model of [3] written in Sec. VD of that paper. We first rewrite Eq. (50) using the unscaled time $t$ and the substitutions $W \rightarrow(D-1) / \sqrt{\epsilon}$ and $\nabla_{\epsilon}^{2} \rightarrow \nabla^{2} / \epsilon$, obtaining

$$
\begin{aligned}
\partial_{t} E= & \frac{i \nabla^{2} E}{1+\epsilon / \Gamma(1)}+\epsilon\left[(1-i \alpha)(D-1)+\frac{\epsilon^{2}(\Delta+\alpha)^{2}}{\Gamma(1)^{2}\left(1+\alpha^{2}\right)}\right. \\
& \left.-\frac{\left(\epsilon \Delta+\nabla^{2}\right)^{2}}{\Gamma(1)^{2}(1-i \alpha)}\right] E,
\end{aligned}
$$

$$
\partial_{t} D=-\sigma \epsilon^{2}\left(D+|E|^{2}-\mu\right) .
$$

Alternatively, if we remove the oscillating term $-i \sigma \eta \psi$, neglect carrier diffusion, and apply the limit $\sigma, a, \eta=O(\epsilon)$, we can write the $\mathrm{CSH}$ model of [3] as

$$
\begin{aligned}
& \partial_{t} \psi= \frac{i a \nabla^{2}}{1+\sigma} \psi+\sigma[(r-1)(1-k-u)-(1+i \alpha) n-(\eta \\
&\left.\left.+a \nabla^{2}\right)^{2}\right] \psi, \\
& \\
& \partial_{t} n=-b n+|\psi|^{2},
\end{aligned}
$$

where $k$ and $u$ are coefficients related to the semiconductor susceptibility and terms of fourth order or higher in $\epsilon$ have been neglected. With the substitutions $\sigma \rightarrow \epsilon, b \rightarrow \sigma \epsilon^{2}, r$ $\rightarrow \mu, \quad \psi \rightarrow \sqrt{b} E, \quad n \rightarrow \mu-D, \quad \eta \rightarrow \epsilon \Delta$, and $\alpha \rightarrow-\alpha$, and a change in the reference frequency, Eq. (C2) becomes

$$
\begin{gathered}
\partial_{t} E=\frac{i a \nabla^{2}}{1+\epsilon} E+\epsilon[(1-i \alpha)(D-1)-(\mu-1)(k+u) \\
\left.-\left(\epsilon \Delta+a \nabla^{2}\right)^{2}\right] E, \\
\partial_{t} D=-\epsilon^{2} \sigma\left(D+|E|^{2}-\mu\right) .
\end{gathered}
$$

The structure of Eq. (C3) is very similar to that of Eq. (C1). The second terms inside the square brackets play a similar role: since we are considering the limit $\mu=1+O\left(\epsilon^{2}\right)$, they introduce a correction of the order $\epsilon^{2}$ in the laser threshold. However, a relevant difference is present in the coefficient of the third terms inside the square brackets, which contain the mechanism of wave-vector selection. The coefficient is equal to 1 in Eq. (C3) and complex in Eq. (C1).
[1] G.-L. Oppo, G. D’Alessandro, and W. J. Firth, Phys. Rev. A 44, 4712 (1991).

[2] J. Lega, J. V. Moloney, and A. C. Newell, Phys. Rev. Lett. 73, 2978 (1994).

[3] J.-F. Mercier and J. V. Moloney, Phys. Rev. E 66, 036221 (2002).

[4] S. Barland, J. R. Tredicce, M. Brambilla, L. A. Lugiato, S. Balle, M. Giudici, T. Maggipinto, L. Spinelli, G. Tissoni, T. Knödl, M. Miller, and R. Jäger, Nature (London) 419, 699 (2002).

[5] X. Hachair, F. Pedaci, E. Caboche, S. Barland, M. Giudici, J. R. Tredicce, F. Prati, G. Tissoni, R. Kheradmand, L. A. Lugiato, I. Protsenko, and M. Brambilla, IEEE J. Sel. Top. Quantum Electron. 12, 339 (2006).

[6] J. Carr, Applications of Centre Manifold Theory (Springer, New York, 1981).

[7] G.-L. Oppo and A. Politi, Z. Phys. B: Condens. Matter 59, 111
(1985); Europhys. Lett. 1, 549 (1986).

[8] P. K. Jakobsen, J. V. Moloney, A. C. Newell, and R. Indik, Phys. Rev. A 45, 8129 (1992).

[9] G. K. Harkness, W. J. Firth, J. B. Geddes, J. V. Moloney, and E. M. Wright, Phys. Rev. A 50, 4310 (1994).

[10] J. Lega, J. V. Moloney, and A. C. Newell, Physica D 83, 478 (1995).

[11] A. C. Newell and J. V. Moloney, Nonlinear Optics, Advanced Topics in Interdisciplinary Mathematical Sciences (AddisonWesley, Reading, MA, 1992).

[12] P. K. Jakobsen, J. Lega, Q. Feng, M. Staley, J. V. Moloney, and A. C. Newell, Phys. Rev. A 49, 4189 (1994).

[13] F. Prati and L. Columbo, Phys. Rev. A 75, 053811 (2007).

[14] F. Pedaci, S. Barland, E. Caboche, P. Genevet, M. Giudici, J. R. Tredicce, T. Ackemann, A. J. Scroggie, W. J. Firth, G.-L. Oppo, and G. Tissoni, Appl. Phys. Lett. 92, 011101 (2008).

[15] K. Staliunas, Phys. Rev. A 48, 1573 (1993). 